any antithrombotic approach, the difficulty is finding efficacy without tipping the hemostatic balance to increased bleeding risk. Indeed, as a player in primary hemostasis, the vWF/platelet GP-Ib-IX axis has, to date, been a challenge to target without considerable risk. The findings by Casari et al. suggest an antithrombotic pathway mechanistic approach that has yet to be considered. While antagonizing the ligand/ receptor interaction could be justified for interrupting primary hemostasis, a type $2 \mathrm{~B}$ mimetic has potential to bind to normal platelet GP-Ib-IX and alter signaling pathways. Could such an approach lead to an antithrombotic effect beyond just blocking a ligand/receptor interaction? Could such a strategy lead to inhibition of activation pathways that mirror aspirin and/ or be complementary? While exploring this strategy is beyond the scope of the study by Casari et al., the identification of altered signaling as a consequence of mutant vWF binding to platelets suggests such an approach might be feasible. Casari and colleagues are to be commended for providing important data to explain molecular consequences for a gain-of-function mutation in vWF many years after the recognition of type $2 \mathrm{~B}$ as a distinct subtype of vWD.

\section{Acknowledgments}

I would like to thank Ginell Post and K.I. Varughese (University of Arkansas for Medical Sciences) for critical comments and helpful discussions.

Address correspondence to: Jerry Ware, University of Arkansas for Medical Sciences, 4301 W. Markham St., Little Rock, Arkansas 72205, USA. Phone: 501.526.6096; Fax: 501.686.8167; E-mail: jware@uams.edu.

1. Ruggeri ZM, Zimmerman TS. von Willebrand factor and von Willebrand disease. Blood. 1987;70(4):895-904.

2. Ruggeri ZM, Mendolicchio GL. Adhesion mechanisms in platelet function. Circ Res. 2007;100(12):1673-1685

3. James PD, Lillicrap D. The molecular characterization of von Willebrand disease: good in parts. $\mathrm{Br} \mathrm{J}$ Haematol. 2013;161(2):166-176.

4. Lopez JA, Andrews RK, Afshar-Kharghan V, Berndt MC. Bernard-Soulier syndrome. Blood.
1998;91(12):4397-4418

5. Ruggeri ZM, Pareti FI, Mannucci PM, Ciavarella N, Zimmerman TS. Heightened interaction between platelets and Factor VIII/von Willebrand factor in a new subtype of von Willebrand's disease. $N$ EnglJ Med. 1980;302(19):1047-1051.

6. Ware J, et al. Identification of a point mutation in type IIB von Willebrand disease illustrating the regulation of von Willebrand factor affinity for the platelet membrane glycoprotein Ib-IX receptor. Proc Natl Acad Sci U S A. 1991;88(7):2946-2950.

7. Celikel R, Ruggeri ZM, Varughese KI. von Willebrand factor conformation and adhesive function is modulated by an internalized water molecule. Nat Struct Biol. 2000;7(10):881-884.

8. Casari C, et al. von Willebrand factor mutation promotes thrombocytopathy by inhibiting integrin $\alpha I I b \beta 3$. J Clin Invest. 2013;123(12):5071-5081.

9. Murata M, Ware J, Ruggeri ZM. Site-directed mutagenesis of a soluble recombinant fragment of platelet glycoprotein Ib $\alpha$ demonstrating negatively charged residues involved in von Willebrand factor binding. J Biol Chem. 1991;266(23):15474-15480.

10. Othman M, Lopez JA, Ware J. Platelet-type von Willebrand disease update: the disease, the molecule and the animal model. Expert Rev Hematol. 2011;4(5):475-477.

11. Guerrero JA, et al. Visualizing the von Willebrand factor/glycoprotein Ib-IX axis with a platelettype von Willebrand disease mutation. Blood. 2009;114(27):5541-5546.

12. Huizinga EG, et al. Structures of glycoprotein Ib $\alpha$ and its complex with von Willebrand factor A1 domain. Science. 2002;297(5584):1176-1179.

\title{
BAFF-ling autoantibodies
}

\author{
Stefanie Sarantopoulos ${ }^{1}$ and Maureen A. Su²
}

1Department of Medicine, Division of Hematological Malignancies and Cellular Therapy, School of Medicine, Duke University, Durham, North Carolina, USA. ${ }^{2}$ Department of Pediatrics, Department of Microbiology and Immunology, School of Medicine, University of North Carolina at Chapel Hill, Chapel Hill, North Carolina, USA.

\begin{abstract}
There is emerging evidence that autoantibodies directed against cytokines modulate the severity of autoimmune disease. Identification of cytokine-targeted autoantibodies in patients can be informative for diagnosis and predicting clinical outcome. In this issue of the JCI, Price and colleagues used a multiplex protein microarray to identify autoantibodies in serum from SLE patients. They found autoantibodies directed against the $B$ cell-activating factor (BAFF) were associated with greater disease severity. This study highlights the contribution of cytokine-directed autoantibodies in disease and describes a valuable tool for identifying autoantibodies against serum antigens.
\end{abstract}

\section{Cytokine-targeting autoantibodies in disease}

Spontaneous autoantibody production is a hallmark of many autoimmune diseases, and these disease-specific autoantibodies are often useful in affirming a clinical autoimmune diagnosis. For instance, clinicians

Conflict of interest: The authors have declared that no conflict of interest exists.

Citation for this article: J Clin Invest. 2013; 123(12):5006-5008. doi:10.1172/JCI73166. test for autoantibodies against insulin and other pancreas-specific antigens to differentiate autoimmune (type 1) diabetes from other types of diabetes. In addition to serving as markers for autoimmunity, autoantibodies can play a central role in disease pathogenesis. Strong evidence exists that autoantibodies are important in development of SLE, a systemic autoimmune disease characterized by immune-mediated injury that affects a large number of tissues, including brain, blood vessels, and kidneys. Autoantibodies against nuclear antigens are important in disease initiation, directly mediating organ injury via complement-mediated cascades and other inflammatory mechanisms.

Interestingly, cytokines have now been described as autoantibody targets in a number of disease settings, resulting in a range of clinical manifestations (1). In many of these diseases, autoantibodies are produced against cytokines that are important in host defense and thus lead to functional immunodeficiency. Autoimmune polyendocrinopathy syndrome type 1 (APS-1), for example, is characterized by multiorgan autoimmunity and mucocutaneous candidiasis (2). For many years, the predisposition to candidiasis, which seemed to reflect a state of immunodeficiency, appeared to be an inconsistent finding among the multiple autoimmune manifestations in APS-1. A possible explanation for this seemingly irregular finding was 


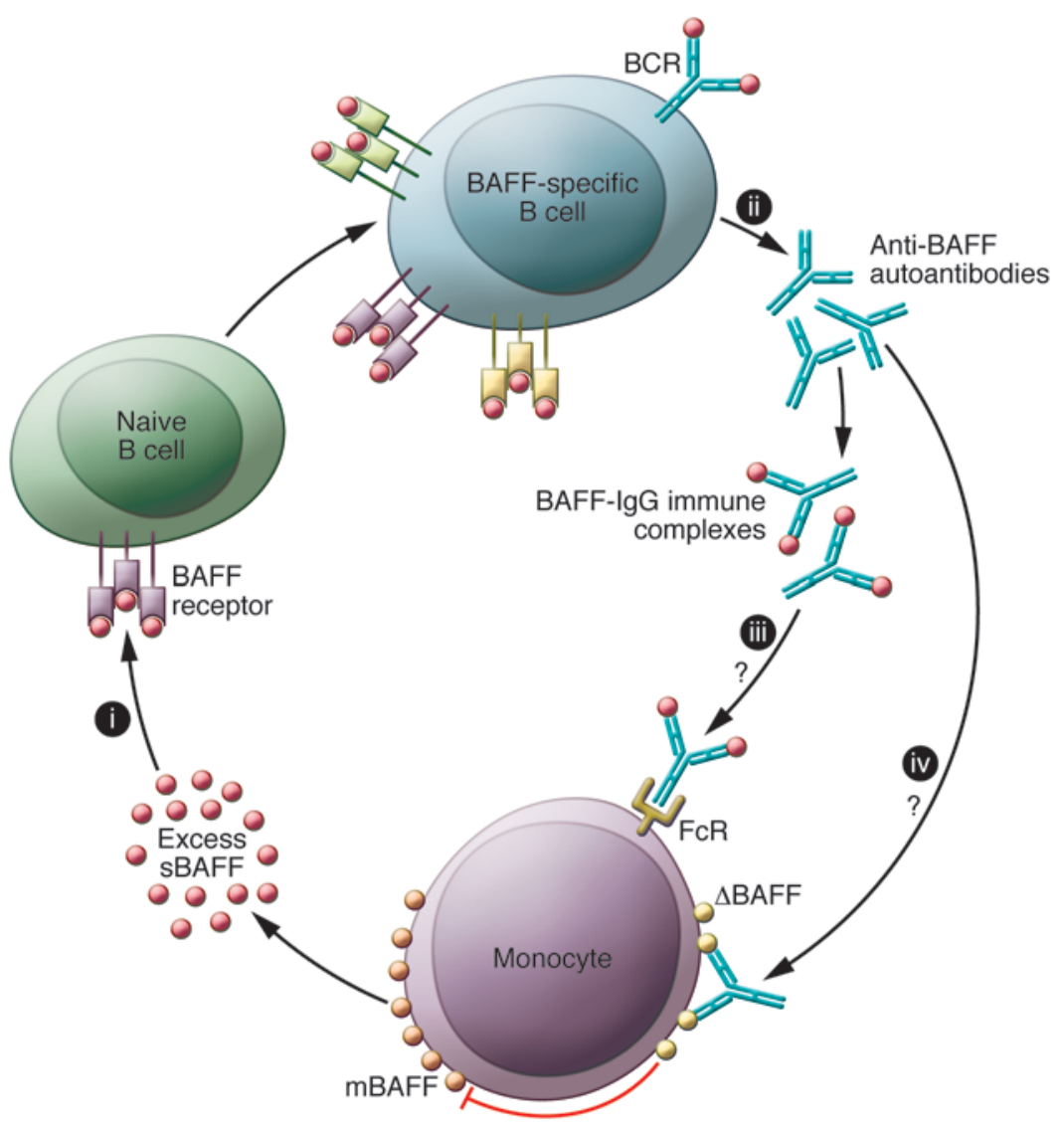

Figure 1

Model for how autoantibodies against BAFF may exacerbate SLE. Excess sBAFF promotes outgrowth of autoreactive B cells, which include BAFF-specific B cells (i). sBAFF (red) may then bind to BAFF-specific $B$ cell receptors (BCR) on $B$ cells and promote production of autoantibodies against BAFF (ii). BAFF-IgG immune complexes may be bound by monocyte Fc receptors $(F c R)$, which stimulate cleavage of membrane BAFF (mBAFF; orange) and further production of SBAFF (iii). In addition, autoantibodies against BAFF may neutralize $\triangle B A F F$ (yellow), a natural regulator of BAFF (iv). Excess sBAFF may then promote autoimmunity and provide further antigen targets for BAFF-specific B cells.

recently revealed by reports of neutralizing autoantibodies against IL-17A and related cytokines in APS- 1 patients $(3,4)$. The activity of IL-17 is essential for host defense against candidal infections $(5,6)$; therefore, autoantibodies that neutralize IL-17 function may predispose APS-1 patients to mucocutaneous candidiasis $(5,6)$. These data indicate that mucocutaneous candidiasis in APS-1 may not reflect primary immunodeficiency, but rather may reflect a primary autoimmunity that secondarily results in immunodeficiency. Other immunodeficient states in which neutralizing autoantibodies against cytokines predispose to infection have been described. These include opportunistic infections with nontuberculous mycobacteria as a result of autoantibodies against $\operatorname{IFN}-\gamma(7,8)$ and staphylococcal skin infections associated with autoantibodies against IL-6 (9). Cytokine-targeting autoantibodies are proving to be a recurring cause of immunodeficiency in various disease settings.

\section{Cytokine-specific autoantibodies modulate disease severity}

In addition to causing immunodeficiency, autoantibodies against cytokines may modulate the severity of autoimmune disease. Approximately $25 \%$ of SLE patients produce autoantibodies against IFN- $\alpha$ (10), a cytokine that is increased in SLE patients and implicated in pathogenesis. IFN- $\alpha$-targeted autoantibodies neutralize IFN- $\alpha$ activity and appear to be associated with milder clinical SLE symptoms. These findings suggest that autoantibody neutralization of IFN- $\alpha$ may alleviate SLE severity, although further work is required to establish causation. In this issue, Price et al. identified autoantibodies against the unique TNF family member B cellactivating factor (BAFF; also called BLys) in SLE patient serum and demonstrated that these autoantibodies neutralize BAFF activity in cell culture systems (11). Furthermore, the authors reported a positive correlation between the presence of neutralizing autoantibodies against BAFF and features associated with a more severe SLE disease profile (11).

At first glance, this finding is somewhat surprising, since studies in mice and humans have shown that BAFF is crucial for SLE pathogenesis. BAFF is a B cell survival factor that is primarily produced by innate immune cells such as monocytes, dendritic cells, neutrophils, and stromal cells, including central nervous system astrocytes and airway epithelia (12). BAFF is produced as a membrane-bound protein that is released following protease cleavage. SLE patients have high circulating levels of BAFF, which correlate with increased autoantibody titers and SLE activity (13, 14). Additionally, the amount of available soluble BAFF (sBAFF) is a pivotal determinant of $B$ cell homeostasis, and BAFF overexpression in mice leads to an autoimmune disease reminiscent of SLE (15, 16). Together, these findings predict that neutralization of BAFF by autoantibodies would be associated with milder disease, rather than severe disease, as observed in this study. One caveat to consider is that Price et al. found this correlation in a small cohort of SLE patients (11); therefore, these results will require validation in larger, independent SLE cohorts. It is possible that these BAFF-targeted antibodies are merely a reflection of an autoimmune response to high amounts of a protein in the circulation, and not involved in disease pathogenesis. If this association between disease severity and the presence of antiBAFF autoantibodies is validated in larger studies, then the question of how a neutralizing autoantibody against a pathogenic cytokine worsens clinical disease will need to be addressed, and the answer will affect current understanding of SLE disease mechanisms.

One possible explanation is that autoantibodies against BAFF paradoxically stimulate sBAFF production (Figure 1). The amount of sBAFF dictates the threshold for negative selection of autoreactive $B$ 
cell clones (17), and excess sBAFF in SLE patients may promote outgrowth of autoreactive $B$ cell clones, including $B$ cells reactive against BAFF itself. Since release of sBAFF results from immune complex activation of monocytes (18), perhaps the formation of BAFF immune complexes induces a positive feedback loop that further increases the amount of available sBAFF. Alternatively, if the anti-BAFF antibodies preferentially bind to a dominant-negative form of BAFF, called $\triangle \mathrm{BAFF}$ (19), the pathologic effects of sBAFF may be unleashed (Figure 1). $\triangle \mathrm{BAFF}$ is the only known natural regulator of $\mathrm{BAFF}$ and is a plausible autoantibody target, since it is a conserved alternate splice isoform.

\section{Implications and future directions}

The presence of endogenous BAFF-specific autoantibodies in patients has potential clinical implications, since a therapeutic antibody against BAFF, belimumab, has recently been FDA approved for treatment of SLE (20). While belimumab has shown efficacy in autoimmune diseases, responses are incomplete, and its effect on the peripheral B cell pool in these patients is unclear. The complexity of BAFF biology and pathology will require further study if we are to effectively implement agents that target this cytokine.

Given the emerging number of anticytokine antibodies being identified, other "immune-modifying" autoantibodies likely exist. Price et al. describe an elegant methodology for screening patient sera using a multiplex protein microarray that will enable discovery of pathophysiologically relevant autoantibodies (11). They validated this platform in patient cohorts of 2 diseases in which autoantibodies against cytokines are a well-described feature: APS-1 and chronic mycobacterial infection (11). Although other multiplexed detection systems are available (21), the approach used by Price et al. provides the benefit of minimizing serum sample volume and increasing the number of cytokine targets that can be screened in parallel (151 antigen targets in this study; ref. 11). Notably, this approach is not limited to detection of autoantibodies against cytokines. Autoantibodies against growth factors, chemokines, and other serum proteins can also be screened on this platform. Low-level reactivity against human growth hormone that was noted in SLE patients using this method (11) may represent one such tantalizing potential autoantibody-based mechanism that underlies SLE clinical findings. Thus, this platform represents a valuable new tool by which to identify autoantibodies against serum antigens and promises to shed light on a wide array of disease mechanisms.

\section{Acknowledgments}

This work was supported in part by NIH grant K08HL107756 (to S. Sarantopoulos) and by a Doris Duke Clinical Scientist Development Award and NIH grant R01 NS079683 (to M.A. Su).

Address correspondence to: Maureen A. Su, University of North Carolina, Chapel Hill, 4123 Thurston Building, CB\#7039, Chapel Hill, North Carolina 27599, USA. Phone: 919.962.2796; Fax: 919.843.7588; E-mail: masu@email.unc.edu.

1. Browne SK, Holland SM. Immunodeficiency secondary to anticytokine autoantibodies. Curr Opin Allergy Clin Immunol. 2010;10(6):534-541.

2. Perheentupa J. Autoimmune polyendocrinopathycandidiasis-ectodermal dystrophy. J Clin Endocrinol Metab. 2006;91(8):2843-2850.

3. Kisand K, et al. Chronic mucocutaneous candidiasis in APECED or thymoma patients correlates with autoimmunity to Th17-associated cytokines. J Exp Med. 2010;207(2):299-308.

4. Puel A, et al. Autoantibodies against IL-17A, IL-17F, and IL-22 in patients with chronic mucocutaneous candidiasis and autoimmune polyendocrine syndrome type I. J Exp Med. 2010; 207(2):291-297.
5. Puel A, et al. Chronic mucocutaneous candidiasis in humans with inborn errors of interleukin-17 immunity. Science. 2011;332(6025):65-68.

6. Milner JD, et al. Impaired $\mathrm{T}_{\mathrm{H}} 17$ cell differentiation in subjects with autosomal dominant hyper-IgE syndrome. Nature. 2008;452(7188):773-776.

7. Höflich C, et al. Naturally occurring anti-IFN- $\gamma$ autoantibody and severe infections with Mycobacterium cheloneae and Burkholderia cocovenenans. Blood. 2004;103(2):673-675.

8. Döffinger R, et al. Autoantibodies to interferon-gamma in a patient with selective susceptibility to mycobacterial infection and organ-specific autoimmunity. Clin Infect Dis. 2004;38(1):e10-4.

9. Puel A, et al. Recurrent staphylococcal cellulitis and subcutaneous abscesses in a child with autoantibodies against IL-6. J Immunol. 2008; 180(1):647-654.

10. Morimoto AM, et al. Association of endogenous anti-interferon- $\alpha$ autoantibodies with decreased interferon-pathway and disease activity in patients with systemic lupus erythematosus. Arthritis Rheum. 2011;63(8):2407-2415.

11. Price JV, et al. Protein microarray analysis reveals BAFF-binding autoantibodies in systemic lupus erythematosus. J Clin Invest. 2013; 123(12):5135-5145.

12. Mackay F, Schneider P. Cracking the BAFF code. Nat Rev Immunol. 2009;9(7):491-502.

13. Zhang J, et al. Cutting edge: a role for B lymphocyte stimulator in systemic lupus erythematosus. J Immunol. 2001;166(1):6-10.

14. Cheema GS, Roschke V, Hilbert DM, Stohl W. Elevated serum B lymphocyte stimulator levels in patients with systemic immune-based rheumatic diseases. Arthritis Rheum. 2001;44(6):1313-1319.

15. Mackay F, et al. Mice transgenic for BAFF develop lymphocytic disorders along with autoimmune manifestations. JExp Med. 1999;190(11):1697-1710.

16. Gross JA, et al. TACI and BCMA are receptors for a TNF homologue implicated in B-cell autoimmune disease. Nature. 2000;404(6781):995-999.

17. Miller JP, Stadanlick JE, Cancro MP. Space, selection, and surveillance: setting boundaries with BLyS. J Immunol. 2006;176(11):6405-6410.

18. Li X, et al. Immune opsonins modulate BLyS/BAFF release in a receptor-specific fashion. JImmunol. 2008; 181(2):1012-1018.

19. Gavin AL, et al. $\delta B A F F$, a splice isoform of BAFF, opposes full-length BAFF activity in vivo in transgenic mouse models. J Immunol. 2005; 175(1):319-328

20. Navarra SV, et al. Efficacy and safety of belimumab in patients with active systemic lupus erythematosus: a randomised, placebo-controlled, phase 3 trial. Lancet. 2011;377(9767):721-731.

21. Ding L, Mo A, Jutivorakool K, Pancholi M, Holland SM, Browne SK. Determination of human anticytokine autoantibody profiles using a particle-based approach. J Clin Immunol. 2012;32(2):238-245. 